\title{
Desenvolvendo estratégia de educação permanente em saúde e reconstruindo práticas em unidade de terapia renal substitutiva
}

\author{
Ana Paula Freitas Lima ${ }^{1}$ \\ Ida Helena Carvalho Francescantonio Menezes $^{2}$ \\ Bárbara Souza Rocha ${ }^{3}$ \\ Edna Regina Silva Pereira ${ }^{4}$
}

\section{RESUMO}

O objetivo deste estudo foi descrever e analisar o desenvolvimento de uma estratégia de educação permanente em saúde na unidade de terapia renal substitutiva utilizando a metodologia da problematização. Pesquisa qualitativa, do tipo pesquisa-ação, embasada na metodologia da problematização, como proposta no Arco de Maguerez. No desenvolvimento das atividades, os participantes definiram como problema a insuficiência de padronização de atendimento multiprofissional ao paciente em terapia renal substitutiva. Os pontos-chave a serem investigados, tais como experiências de outras instituições, organização da linha de cuidados à luz da legislação e competências específicas próprias dos profissionais componentes da equipe, efetuaram-se na etapa de teorização. Posteriormente foram formuladas hipóteses de solução como estabelecimentos de protocolos, construção de termo de consentimento livre e esclarecido e realização de reuniões multiprofissionais para discussão de casos e definição de plano de cuidado coletivo. As propostas de solução mais viáveis foram selecionadas para serem aplicadas na prática e ajudar na superação do problema. Os participantes puderam vivenciar a metodologia da problematização, refletir sobre suas práticas, praticar a tomada de decisão compartilhada na unidade com base na viabilidade das propostas, permitindo avanços repensando e reconstruindo a realidade para melhoria dos serviços oferecidos por meio da educação permanente em saúde.

Palavras-chave: Educação continuada. Capacitação de recursos humanos em saúde. Terapia de substituição renal.

\section{Rebuilding practices in renal replacement terapy unit through the problematization methodology}

\footnotetext{
ABSTRACT

The aim of this study was to describe and analyze the developing of a health permanent education strategy in renal replacement therapy unit using the problematization methodology. Qualitative research, of the research-action type, based on the problematization methodology, as proposed in Maguerez's Arc. In the development of activities, participants defined as a problem the insufficient

${ }^{1}$ Enfermeira. Mestrado em Ensino na Saúde. Hospital das Clínicas da Universidade Federal de Goiás (HC/UFG). E-mail: anapflima@ufg.br

${ }^{2}$ Nutricionista. Doutorado em Ciências da Saúde. Professora Adjunta da Faculdade de Nutrição da Universidade Federal de Goiás. E-mail: idahelenamenezes@gmail.com

${ }^{3}$ Enfermeira. Doutorado em Enfermagem. Professora Assistente da Faculdade de Enfermagem da Universidade Federal de Goiás. E-mail: barbarasrocha@gmail.com

${ }^{4}$ Médica. Pós-doutorado em Educação Médica. Professora Titular da Faculdade de Medicina da Universidade Federal de Goiás. E-mail: ersp13@gmail.com
} 


\section{Programa de Pós-Graduação \\ em Ensino na Saúde - MEPES/UFG \\ DOSSIÊ \\ ENSINO NA SAÚDE}

ITINERARIUS

REFLECIONIS

V.17, N.01, 2021. ISSN: 1807-9342

standardization of multidisciplinary care to patients on renal replacement therapy. The key points to be investigated, such as the experiences of other institutions, organization of care line in light of the legislation and own specific skills of team members professionals, promoted the theory stage. Later were formulated possible solutions as protocols establishments, construction of a free informed consent term and implementation of multidisciplinary meetings to discuss cases and definition of collective care plan. The most viable solution proposals were selected to be applied in practice and help in overcoming the problem. Participants were able to experience the problematization methodology, reflect on their practices, practice the shared collective decision making in the unit based on the feasibility of the proposals, allowing advances in rethinking and rebuilding the reality to improve the services offered through health permanent education.

Key-words: Continuing education. Health human resource training. Renal replacement therapy.

\section{INTRODUÇÃO}

No século XXI, a Doença Renal Crônica (DRC) tem se apresentado como problema mundial de saúde pública, pelos aumentos significativos de suas taxas de incidência, prevalência, morbidade e mortalidade. A grande maioria dos pacientes que alcançam estágio avançado da doença renal estão em tratamento dialítico, com prevalência de 280 casos por milhão de pessoas, em comparação com 65 casos por milhão de pessoas que vivem com um rim transplantado (WEBSTER et al., 2017). No Brasil, de acordo com o censo realizado pela Sociedade Brasileira de Nefrologia, no ano de 2017, o número total estimado de pacientes em tratamento dialítico no país era de 126.583 , representando um aumento de 3.753 pacientes (3\%) em um ano (THOMÉ et al., 2019).

A Portaria $n^{\circ} 389$, de 13 de março de 2014, estabelece que os serviços de saúde integrantes da linha de cuidado à pessoa com DRC deverão observar a garantia de Educação Permanente em Saúde (EPS) de profissionais da saúde para a prevenção, diagnóstico e tratamento da DRC e dos fatores de risco que levam à DRC, de acordo com as diretrizes da Política Nacional de Educação Permanente (ANVISA, 2014).

A Política Nacional de Educação Permanente de Saúde (PNEPS) proposta pelo Ministério da Saúde, a define como:

A Educação Permanente é aprendizagem no trabalho, onde o aprender e o ensinar se incorporam ao quotidiano das organizações e ao trabalho. Propõe-se que os processos de capacitação dos trabalhadores em saúde tomem como referências as necessidades de saúde das pessoas e das populações, da gestão setorial e do controle 


\section{Programa de Pós-Graduação \\ em Ensino na Saúde - MEPES/UFG \\ DOSSIÊ \\ ENSINO NA SAÚDE}

ITINERARIUS

REFLECIONIS

V.17, N.01, 2021. ISSN: 1807-9342

social em saúde, tenham como objetivos a transformação das práticas profissionais e da própria organização de trabalho (BRASIL, 2004, p.38).

Essa política possibilitou o desenvolvimento de espaços, onde é possível se analisar a prática cotidiana dentro dos serviços de saúde, com o objetivo de transformá-la. Portanto, considerando a importância da terapia renal substitutiva (TRS) no contexto da saúde pública, é necessário que esses serviços de saúde conheçam as práticas de sua equipe e implementem estratégias de EPS com a finalidade de aproximar da sua realidade e contribuir para a qualidade da assistência (BRASIL, 2004; GIGANTE; CAMPOS, 2016).

Estudo de revisão integrativa de literatura evidenciou diversas publicações que retrataram a EPS vinculada a momentos assistemáticos, não contínuos, com perfil de ações educativas distante da concepção de integralidade com base tecnicista, demonstrando fragilidade da abordagem nos processos educativos dos profissionais verdadeiramente articulados ao processo de trabalho. Houve destaque, porém, nas publicações de experiências nos serviços de saúde no Brasil que contemplaram o princípio preconizados pela PNEPS, com concepção problematizadora nas ações educativas desde seu planejamento, execução e avaliação (CAMPOS; SENA; SILVA, 2017).

As tendências pedagógicas que direcionam o processo de ensino-aprendizagem, partindo do pressuposto que a interação entre o sujeito e o processo de aprendizagem está intermediado por estruturas cognitivas e concepções em relação a inserção social dos indivíduos e as suas formas de aprender, devem ser tomadas como um elemento fundamental desse processo (CONTERNO; LOPES, 2016).

Desse modo, a EPS vem pautada na concepção pedagógica transformadora e emancipatória de Paulo Freire, que chama a atenção para o processo de ensinar e aprender, propondo reflexão sobre as formas de abordagem, a partir do conhecimento prévio e protagonismo dos educandos, com o objetivo de aprendizagem nos cenários de atenção à saúde e como capazes de provocar transformações de práticas pedagógicas e assistenciais (CECCIM; CYRINO, 2017). 


\section{Programa de Pós-Graduação \\ em Ensino na Saúde - MEPES/UFG \\ DOSSIÊ \\ ENSINO NA SAÚDE}

ITINERARIUS

REFLECIONIS

V.17, N.01, 2021. ISSN: 1807-9342

Para tanto, a metodologia da problematização (MP) caracteriza-se como metodologia de ensino, estudo e trabalho, a qual pode ser utilizada para promover a mobilização do potencial dos educandos por meio de um processo que envolve ação-reflexão sobre um aspecto da realidade observada, em situações em que os temas estejam relacionados com a vida em sociedade (BERBEL, 1998; BERBEL, 2012).

O Arco de Maguerez, base para a aplicação da MP foi elaborado na década de 1970, porém apenas no final dos anos de 1980, com inspirações em Paulo Freire, foi publicado por Bordenave e Pereira (1982) que apresentaram a proposta como um caminho de educação. No século XX com a forte demanda por práticas educativas com o protagonismo dos educandos na construção do conhecimento, o potencial pedagógico dessa metodologia passou a ser considerado de maior relevância (COLOMBO; BERBEL, 2007).

Assim sendo, a MP diferencia-se de outras metodologias por ter como ponto de partida e chegada a realidade a ser problematizada e extrapolar a sua caracterização apenas como método, pelo movimento a ela atribuído que permite enxergar e transformar a realidade com maior criticidade, além de uma prática mais contextualizada, tão necessária a área da saúde. No entanto, para efetividade do processo de ensino-aprendizagem torna-se necessário o cumprimento de todas as etapas do Arco de modo integrado, configurando um ciclo pedagógico e didático com oportunidade de aprendizado a partir da realidade vivida (COLOMBO; BERBEL, 2007; BERBEL; GAMBOA, 2012).

Os profissionais de saúde envolvidos no serviço de TRS precisam ser críticos e reflexivos, estarem preparados para atuar de forma integral e interdisciplinar na atenção ao portador de DRC. Entendendo toda a complexidade do cotidiano da prática nesse tipo de unidade e da instituição onde esse estudo foi desenvolvido, apresentamos a seguinte pergunta de pesquisa: O desenvolvimento de estratégias de EPS com o uso da MP permite o aprimoramento e mudanças na prática dos profissionais de TRS? Nesse sentindo, o objetivo desse estudo foi descrever e analisar o desenvolvimento de uma estratégia de educação permanente em saúde na unidade de terapia renal substitutiva utilizando a metodologia da problematização. 


\section{METODOLOGIA}

Trata-se de uma pesquisa qualitativa, do tipo pesquisa-ação, que utiliza técnicas para obter melhorias nos cenários de práticas por meio de uma abordagem intervencionista e problematizadora na realidade não manipulada (TRIPP, 2005). Além disso, esse método participativo prevê o envolvimento dos participantes na realização de uma ação coletiva, o que inclui a interação com os pesquisadores como sujeitos inseridos no campo de contexto da pesquisa (SILVA et al.,2011). Esse estudo seguiu o check-list do Consolidated criteria for reporting qualitative research (COREQ) que identifica critérios como forma de exploração de estudos qualitativos (TONG; SAINSBURY; CRAIG, 2007).

A pesquisa foi desenvolvida durante o ano de 2017 em um hospital de ensino público federal, que oferece o serviço de TRS nas modalidades de hemodiálise e diálise peritoneal. A população do estudo constitui-se da equipe assistencial de nível superior que desempenha suas funções nesse serviço, totalizando 16 profissionais, que foram recrutados pessoalmente para a participação, sendo excluídos aqueles que se encontraram afastados das atividades devido a férias e/ou licença médica no período de coleta de dados. Destes, 9 aceitaram participar da pesquisa, demonstrando interesse e disponibilidade, além de concordarem com a metodologia adotada. Vale ressaltar que a única categoria profissional de nível superior que compõe a equipe e que não participou do estudo foi o serviço social, por não ter respondido ao recrutamento para participação.

A condução da pesquisa foi mediada pela pesquisadora principal, mestranda em ensino na saúde e profissional do serviço, com apoio das demais pesquisadoras como facilitadoras, sendo todas doutoras, participantes do Programa de Desenvolvimento Docente para Educadores das Profissões da Saúde (FAIMER®) e uma com formação básica em dinâmica de grupo e em coaching.

O método da pesquisa e os dados foram obtidos por meio de observação participante, a partir de encontros embasados na MP, percorrendo as etapas do Arco de 


\section{Programa de Pós-Graduação \\ em Ensino na Saúde - MEPES/UFG \\ DOSSIÊ \\ ENSINO NA SAÚDE}

ITINERARIUS

REFLECIONIS

V.17, N.01, 2021. ISSN: 1807-9342

Maguerez (observação da realidade, pontos-chave, teorização, hipóteses de solução e aplicação à realidade). A MP permite a coleta, registro, organização, sistematização e tratamento de dados, quando percorre suas etapas passando por um processo de reflexão sobre problemas detectados e retorna para a parcela da realidade com uma prática mais consciente $\mathrm{e}$ transformadora (BERBEL; GAMBOA, 2012).

A coleta de dados da pesquisa foi estruturada segundo o esquema apresentado na Figura 1. Foram realizados quatro encontros sendo um preliminar para contato prévio com os participantes e os demais para a problematização em si. Os encontros foram realizados em uma sala na instituição que favorecia a participação e interação, evitando fatores que poderiam desviar a atenção ou interromper a discussão. A duração média de cada encontro foi de duas horas, realizado em horário compatível com a disponibilidade dos profissionais.

Figura 1: Representação esquemática da coleta de dados.

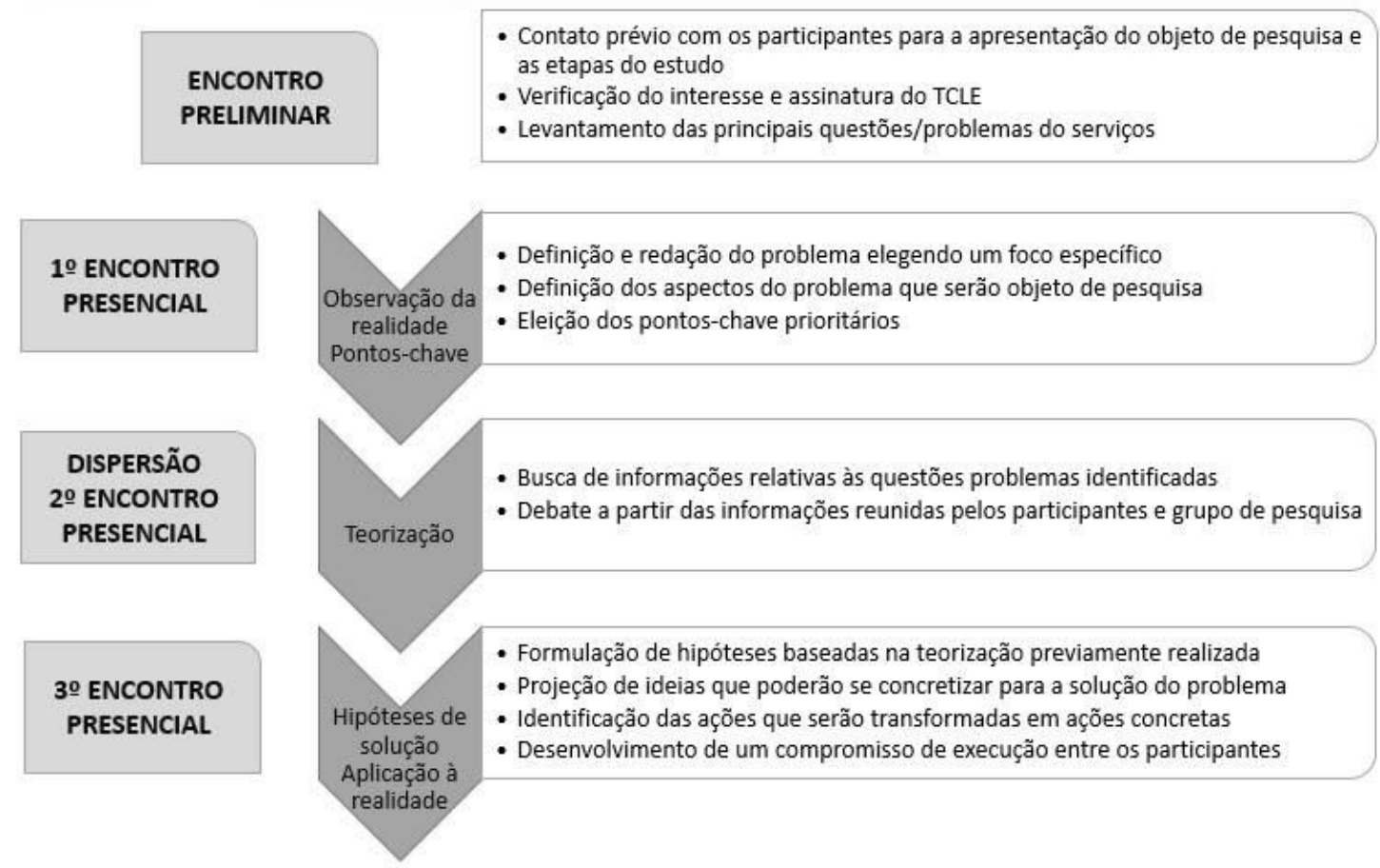

Fonte: elaborado pelas autoras, 2019.

Estavam presentes nos encontros os participantes da pesquisa, as pesquisadoras e contamos com a presença da pedagoga da instituição que compõe o núcleo de EPS. Os dados 


\section{Programa de Pós-Graduação \\ em Ensino na Saúde - MEPES/UFG \\ DOSSIÊ \\ ENSINO NA SAÚdE}

ITINERARIUS

REFLECIONIS

V.17, N.01, 2021. ISSN: 1807-9342

foram registrados em diário de campo com as anotações da dinâmica do grupo e expressões verbais e corporais, assim como as produções derivadas das atividades desenvolvidas durante os encontros. Nessas atividades foram utilizados recursos como targetas e flipchart com intuito de estimular a participação do grupo, assim como diversas técnicas e ferramentas.

No encontro preliminar os participantes, pela observação da realidade do serviço no qual estão inseridos foram instigados pelos pesquisadores a debater as principais situações ou problemas vivenciados com foco no processo de trabalho e necessidade de EPS, seguida de uma seleção de qual seria a situação-problema principal que atenderia a equipe. No $1^{\circ}$ encontro já com o recorte da realidade a ser observado, para o desencadeamento das demais etapas do Arco, foram discutidas as informações observadas na realidade, permitindo que o problema fosse redigido elegendo um foco específico, assim como as justificativas para a escolha do problema e possíveis contribuições para o meio. Na sequência foram levantadas as questões que no entendimento do grupo poderiam ser possíveis razões e dimensões para a existência e manutenção do problema, sendo eleitos os aspectos a serem estudados e investigados por meio da redação dos pontos-chave.

No passo seguinte que consistia em uma etapa de investigação, os participantes tiveram três semanas para buscar, individualmente ou em grupo, informações acerca do problema de fontes variadas e, posteriormente no $2^{\circ}$ encontro foi possibilitado o compartilhamento dessas informações e o estabelecimento de relações entre essas, concluindo a etapa de teorização.

No $3^{\circ}$ encontro presencial, foi retomado todo o caminho percorrido pelo grupo até o momento com o intuito de possibilitar a projeção de ideias que poderiam se concretizar para a solução do problema, levantando as hipóteses de solução, compromissos e encaminhamentos. Foi então executada uma seleção daquelas alternativas de solução apresentadas em relação a aplicabilidade, para a eleição daquelas que poderiam ser transformadas em ações concretas e aplicadas à realidade do serviço.

Os dados foram analisados de acordo com a análise de conteúdo proposta por Bardin (2016), observando as etapas de pré-análise dos dados, exploração e tratamento dos resultados, sendo as categorias emergentes pré-definidas de acordo o esquema de progressão 
pedagógica do Arco de Charles Maguerez, além de núcleos de sentido apresentados no material produzido.

A pesquisa foi desenvolvida seguindo os pressupostos éticos da Resolução 466/2012 (BRASIL, 2012), e só teve início após avaliação e aprovação do Comitê de Ética em Pesquisa do Hospital das Clínicas da Universidade Federal de Goiás, sob parecer $n^{\circ}$ 2.596.347 no dia 29 de agosto de 2017 e todos os participantes assinaram o Termo de Consentimento Livre e Esclarecido (TCLE).

\section{RESULTADOS E DISCUSSÃO}

A partir da identificação de um problema a ser trabalhado pelos participantes, por meio da análise situacional do serviço, diversas ferramentas educacionais e de gestão foram apresentadas nos encontros, no sentido de apoiar a construção de um plano de intervenção aplicável à realidade. Esse plano foi baseado nas hipóteses de solução definidas a partir de um aprofundamento teórico-reflexivo na etapa de teorização sobre as causas e consequências do problema identificado. A escolha da MP tem como diferencial a possibilidade de retorno para a realidade, porém tal desafio, pressupõe domínio teórico-metodológico da concepção de educação problematizadora pelos envolvidos. A Figura 2 demonstra os caminhos percorridos durante a aplicação do Arco de Maguerez na estratégia de EPS.

Figura 2: Execução do Arco de Maguerez em estratégia de Educação Permanente em Saúde em unidade de terapia renal substitutiva. 


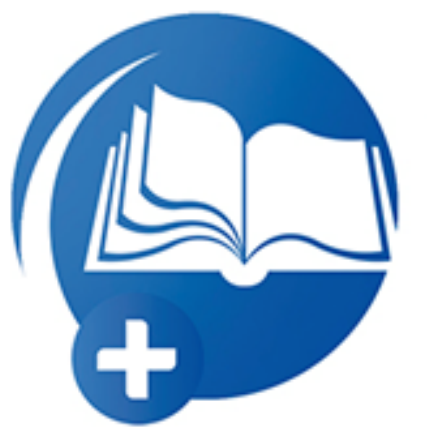

\section{Programa de Pós-Graduação \\ em Ensino na Saúde - MEPES/UFG}

DOSSIÊ

ENSINO NA SAÚdE

ITINERARIUS

REFLECIONIS

V.17, N.01, 2021. ISSN: 1807-9342

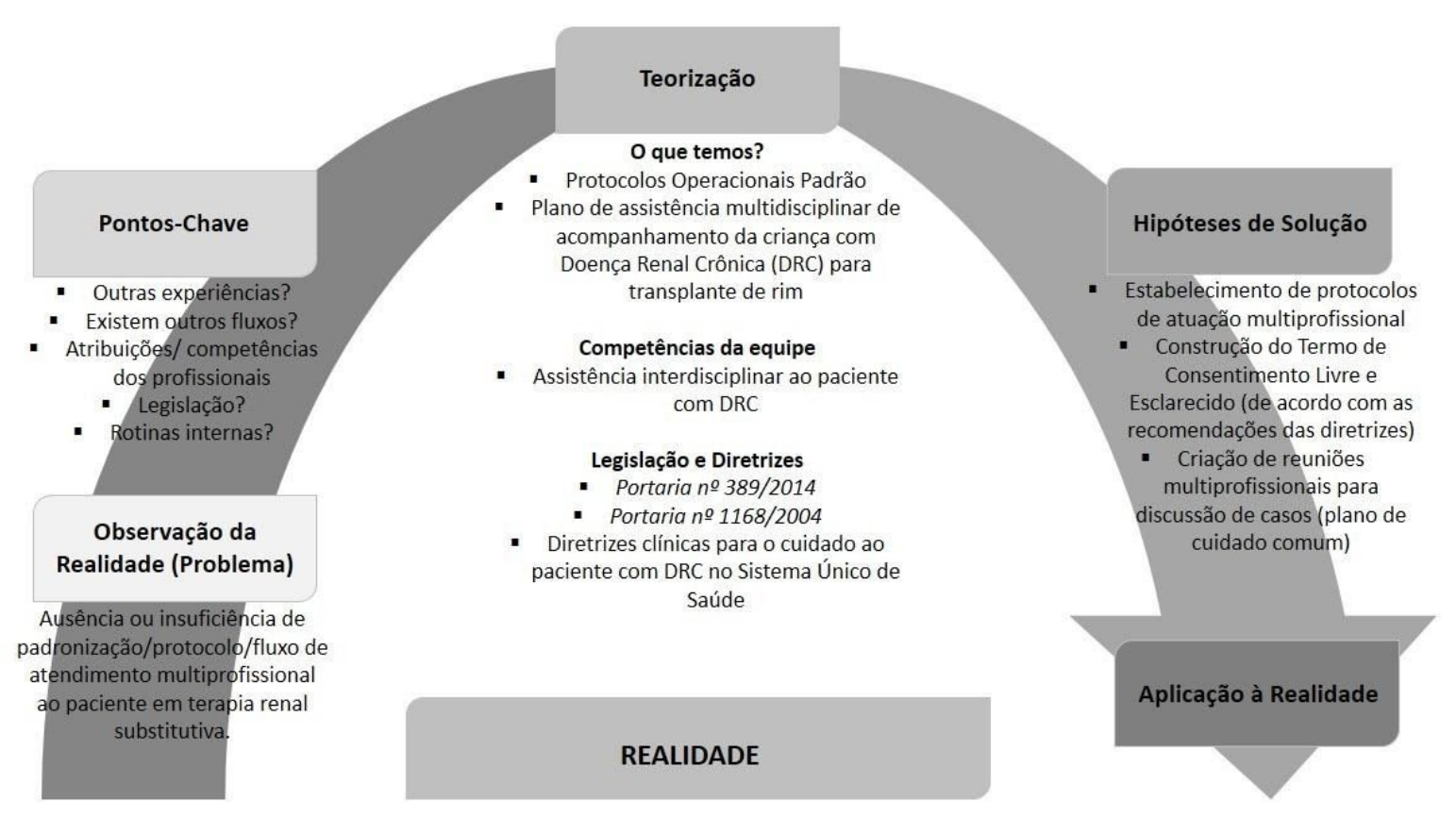

Fonte: elaborado pelas autoras, 2019.

Os participantes da pesquisa compuseram um grupo com idade de 26 a 46 anos, com tempo de formação entre 3 a 20 anos, sendo a maioria mulheres com nível de formação profissional que variou entre especialistas e doutores, composto por três médicos (as) assistenciais, uma médica residente em nefrologia, três enfermeiras, uma psicóloga e uma nutricionista.

\subsection{Observação da realidade: ausência de fluxo de atendimento multiprofissional}

Considerando o contexto atual da realidade na qual se pretendeu intervir (serviço de TRS de um hospital público federal de ensino), os participantes foram convidados a trabalhar com alguns movimentos para a identificação de problemas. No primeiro momento fizeram um levantamento por intermédio da técnica brainstorming de quais eram os desconfortos em relação a realidade do ambiente de trabalho considerados importantes pelo grupo, sendo essas ideias organizadas por um facilitador no flipchart. Seguindo de um 


\section{Programa de Pós-Graduação \\ em Ensino na Saúde - MEPES/UFG \\ DOSSIÊ \\ ENSINO NA SAÚdE}

ITINERARIUS

REFLECIONIS

V.17, N.01, 2021. ISSN: 1807-9342

segundo movimento de identificação individual da situação, onde cada participante elegeu dois problemas ou necessidades em relação ao contexto do mundo do trabalho para o qual gostaria de direcionar a estratégia de EPS, ou seja, a realidade sobre a qual gostaria de intervir. Cada demanda foi expressa em uma tarjeta, sendo duas tarjetas por participante, e fixadas em mural permitindo a visualização de todos os presentes, seguida de um movimento de identificação de conjunto de problemas ou necessidades (técnica de visualização móvel), por meio de esclarecimento das opiniões individuais de forma a permitir um agrupamento de ideias afins percebidas pelo grupo, permitindo a escolha de qual seria o primeiro problema a ser abordado pelo grupo de trabalho.

Colombo e Berbel (2007) afirmam que já nessa primeira etapa, permite-se aos participantes o desenvolvimento de uma postura crítica em relação à realidade, por intermédio de saberes previamente existentes, denominado saber existencial, a eleição de um recorte da realidade e percepção da necessidade de um conhecimento mais especializado que desencadeará as etapas subsequentes.

No chamado primeiro encontro presencial com o apoio dos facilitadores, os participantes redigiram e elegeram o melhor enunciado para o problema, a partir do movimento de observação da realidade na MP. O grupo entendeu que a ausência ou insuficiência de fluxo de atendimento multiprofissional ao paciente em TRS gera impacto diretamente na assistência desses usuários, visto que a dinâmica do trabalho nesses serviços possui caráter fundamental para a possibilidade de melhora clínica desses pacientes. Segundo Hamilton e Sessoms (2011), conhecer o fluxo de trabalho em serviços de diálise é um importante método para identificar causas potenciais e desenvolver soluções adequadas para problemas existentes.

Assim, a constatação pelos participantes que a atuação da equipe multiprofissional contribui para a manutenção da vida dos pacientes em TRS é uma importante característica do processo de trabalho, que pode repercutir positivamente na identidade profissional dos trabalhadores inseridos nesse contexto laboral, para além da fragilidade de rotinas marcadas pela pressão do tempo em relação a disponibilidade de recursos humanos no serviço e a repetição de tarefas. 


\section{Programa de Pós-Graduação \\ em Ensino na Saúde - MEPES/UFG \\ DOSSIÊ \\ ENSINO NA SAÚdE}

ITINERARIUS

REFLECIONIS

V.17, N.01, 2021. ISSN: 1807-9342

Além disso, o problema levantado pelos participantes vai ao encontro das recomendações da literatura em relação ao manejo dos pacientes portadores de DRC e a participação em programas de assistência multiprofissional, que incluem a orientação do paciente e de sua família sobre as modalidades dialíticas, como a hemodiálise e diálise peritoneal, ou transplante renal (SINGH; KARI, 2016). Estudos sugerem que o modelo de atendimento envolvendo equipe multidisciplinar ao oferecer os cuidados necessários, de forma abrangente e organizada, parece ser a melhor forma de tratar a DRC, demonstrando que esses pacientes quando previamente expostos ao atendimento interdisciplinar, antes do início da diálise, apresentavam maior sobrevida, além de uma tendência à diminuição de risco de hospitalização (BASTOS; KIRSZTAJN, 2011; HEMMELGARN et al., 2007; GOLDSTEIN et al., 2004).

Nesse sentido, a EPS tem como base emergir o conhecimento a partir da realidade do serviço, Mehry (2015) utiliza o termo "agir no trabalho vivo em ato" para denominar práticas de saúde com intervenções que permitem ao profissional na produção do cuidado ser o centro do processo com ações que possam efetivamente mudar o modo de fabricar saúde, sendo a realidade o local de formação.

\subsection{Identificação de pontos-chave: outras experiências, fluxos e atribuições}

Nessa experiência, para a definição dos pontos-chave, as facilitadoras suscitaram questionamentos tais como: Quais são os protocolos de atendimento ao portador de DRC em TRS em outros países ou hospitais universitários? O que a nossa legislação traz sobre a equipe e organização da linha do cuidado a pessoas com DRC? Quais as competências especificas próprias dos profissionais de saúde que prestam assistência na TRS? Dentre outros, que contribuíram para a reflexão do tema em questão. Outros estudos relatam a experiência dos tutores ou facilitadores no exercício do seu papel na condução desse processo, a fim de conduzir as discussões e gerenciar o conhecimento que emerge do aprendizado, porém sem tomar o processo de ensino para si (PRADO et al., 2012; MELO et al., 2016). 


\section{Programa de Pós-Graduação \\ em Ensino na Saúde - MEPES/UFG \\ DOSSIÊ \\ ENSINO NA SAÚdE}

ITINERARIUS

REFLECIONIS

V.17, N.01, 2021. ISSN: 1807-9342

Analisando o problema projetado pelos profissionais desse serviço, essa fase permitiu aprofundar a sua percepção e formular um conjunto de prováveis explicações para a sua ocorrência, mediante a identificação dos pontos-chave e as variáveis determinantes da situação, enumerando os aspectos que necessitavam de melhor compreensão para buscar uma resposta ao problema. Na segunda etapa do esquema de progressão pedagógica estruturado pelo Arco de Maguerez, espera-se dos participantes o envolvimento e a iniciativa, além de autonomia, pois nessa etapa permite-se a identificação e questionamento dos possíveis fatores que afetam o contexto no qual o problema ocorre (VIEIRA; PANÚNCIO-PINTO, 2015).

Nessa etapa de identificação de pontos-chave, foi explorado com o grupo de participantes a situação real vivida por eles, com o intuito de perceber suas formas de inserção dentro da equipe e suas respectivas funções. Os participantes perceberam a importância de competências específicas próprias de cada profissional na qualidade do cuidado prestado ao portador de DRC em TRS, porém reconhecem como fundamentais práticas colaborativas para a tomada de decisões relativas à melhoria da assistência prestada.

Esses profissionais apontaram limitações relacionadas ao desconhecimento de experiências de construção de fluxos de trabalho multiprofissional em outros serviços de TRS e a necessidade de estabelecimentos e revisão de rotinas internas, de acordo com as legislações existentes. Sendo assim, após os relatos e discussões os pontos-chave a serem pesquisados pelos participantes foram sintetizados como: outras experiências, existência de outros fluxos, atribuições / competências dos profissionais, legislação e rotinas internas.

\subsection{Teorização: linha do cuidado e assistência ao portador de DRC}

Na terceira etapa, que consiste na teorização, os participantes dispuseram de um tempo de três semanas que atribuímos o nome de dispersão, com a finalidade de instrumentalização teórica para as etapas subsequentes. Nesse período ocorreu a busca de conhecimento e informações em variadas fontes como informação de especialistas, pesquisas de legislações e diretrizes existentes, experiências de protocolos e relatos de outras 


\section{Programa de Pós-Graduação \\ em Ensino na Saúde - MEPES/UFG \\ DOSSIÊ \\ ENSINO NA SAÚDE}

ITINERARIUS

REFLECIONIS

V.17, N.01, 2021. ISSN: 1807-9342

instituições. Essa etapa visa permitir a construção de respostas embasadas na literatura, sendo que as informações coletadas devem estabelecer relação com os pontos-chave identificados anteriormente em função do problema, com o objetivo de transformar a realidade eleita para 0 estudo (COLOMBO; BERBEL, 2007).

No segundo encontro presencial os participantes foram divididos em três grupos de acordo com as informações coletadas, denominados: O que temos (protocolos e planos de assistência multidisciplinar já existentes do serviço), Competência da equipe (assistência interdisciplinar ao paciente com DRC) e Legislações e diretrizes (portarias que definem os critérios para a organização da linha do cuidado ao paciente portador de DRC e as diretrizes clínicas para os cuidados desses pacientes no Sistema Único de Saúde). Nesse momento, os participantes tiveram a oportunidade de partilhar o estudo realizado e discutir as principais informações que poderiam contribuir para a compreensão do problema e projeção de hipóteses de solução, sendo registrada pelos participantes a teorização desenvolvida.

As facilitadoras contribuíram realizando uma preleção sobre a diferenciação conceitual de protocolos, procedimentos operacionais padrão e fluxogramas, assim como trazendo exemplos de fluxos de trabalhos desenvolvidos pelo núcleo interno de regulação da instituição, afim de subsidiar aquisição de novos conhecimentos para transformação da realidade vigente. Outras experiências da utilização da MP relatam o papel dos facilitadores nessa etapa de teorização na instrumentalização dos participantes, tanto na seleção de materiais a serem trabalhados quanto na apresentação de questões relevantes, sendo essas necessárias para a aquisição de novos conhecimentos não contemplados durante a dispersão e pesquisas realizadas pelo grupo (DE PAULA et al., 2013; FUJITA et al., 2016).

O grupo avançou percebendo que alguns documentos existentes no serviço apesar de estarem denominados como, não se enquadram como protocolos que permitem o enfrentamento de problemas assistenciais e de gestão na unidade. $\mathrm{O}$ aparecimento recorrente dos protocolos como base para a transformação da realidade nos relatos dos participantes demonstra a preocupação em melhorar as condições de trabalho e promover um ambiente mais seguro para a assistência aos portadores de DRC em TRS. Porém, é importante salientar que os protocolos, enquanto estratégias fundamentais, tanto no planejamento e implementação 


\section{Programa de Pós-Graduação \\ em Ensino na Saúde - MEPES/UFG \\ DOSSIÊ \\ ENSINO NA SAÚdE}

ITINERARIUS

REFLECIONIS

V.17, N.01, 2021. ISSN: 1807-9342

de ações, quanto na padronização do processo de trabalho, apresentam limitações com risco de produção de processos que não permitam um posicionamento crítico-reflexivo dos profissionais (WERNECK; FARIA; CAMPOS, 2009).

Os participantes mencionaram ainda dificuldades iniciais no desenvolvimento de processos de trabalho de forma multiprofissional, com ausência de clareza do fluxo a ser percorrido e necessidade de definição desse. Nessa etapa de teorização os protocolos foram então, reconhecidos como ferramentas que permitem a orientação de fluxos, condutas e procedimentos. Foi notada grande ansiedade nas falas no que diz respeito à falta de manutenção de atendimento multiprofissional aos usuários com relatos explicitando momentos em que o serviço fica descoberto, apesar da obrigatoriedade, estabelecida pela RDC $n^{\circ} 154$ da ANVISA, de que cada serviço de diálise tenha a ele vinculado um mínimo de dois médicos nefrologistas, dois enfermeiros, um assistente social, um psicólogo e um nutricionista, assim como auxiliares e técnicos de enfermagem de acordo com o número de pacientes (ANVISA, 2004).

\subsection{Hipóteses de solução: protocolos, TCLE e reuniões multiprofissionais}

Nessa etapa, foi proposto aos participantes o levantamento das hipóteses de solução embasado na teorização previamente realizada, tendo em vista o recorte da realidade. Essas escolhas consideraram aspectos subjetivos e objetivos, incluindo informações e percepções que permitiram aos participantes a tomada de decisão, sendo negociada e pactuada pelo grupo. Foram projetadas e registradas diversas hipóteses pelos participantes que poderiam vir a se transformar em ações concretas para solucionar ou caminhar em direção a solução do problema trabalhado nessa estratégia de EPS.

A quarta etapa do Arco de Charles Maguerez é caracterizada pela formulação das hipóteses que deve ser norteada pela percepção do problema e pode abranger diferentes níveis de ação, devendo os participantes explicar e argumentar o sentido das mesmas na elaboração 


\section{Programa de Pós-Graduação \\ em Ensino na Saúde - MEPES/UFG \\ DOSSIÊ \\ ENSINO NA SAÚDE}

ITINERARIUS

REFLECIONIS

V.17, N.01, 2021. ISSN: 1807-9342

de alternativas de solução (COLOMBO; BERBEL, 2007; VILLARDI; CYRINO; BERBEL; 2015).

Na organização do trabalho em equipe os participantes reconhecem que nenhum profissional perde seu campo específico de atuação, porém a ação profissional conjunta permite um modo de abordar e enfrentar os problemas, sendo a equipe, de forma compartilhada, responsável por compreender as demandas dos usuários, propor com a participação desses, modos de atuar nos seus problemas relacionados ao adoecimento crônico. Estudo demonstrando os princípios básicos, passos de otimização do fluxo de trabalho em serviço de diálise e discussão de mudanças afirma que inúmeros fatores devem ser levados em consideração ao desenvolver ou alterar um cronograma de tratamento dos pacientes em TRS, definindo metas e objetivos em busca de qualidade, segurança e satisfação dos pacientes e profissionais (SESSOMS; HAMILTON, 2011).

Além disso, apareceu como resultado nessa etapa questões relacionadas ao estabelecimento de normas, rotinas e regulamentos como necessários para o funcionamento efetivo desses serviços de saúde, corroborando com outro estudo que aponta a implementação/alteração de protocolos como conduta adotada para a prevenção de eventos adversos em serviço de hemodiálise, tornando mais eficiente o processo de trabalho e uniformização da assistência (SOUSA et al.; 2013).

A partir da discussão da solução do problema fundamentada na teorização, foram então definidas as seguintes hipóteses como mais viáveis: estabelecimento de protocolos de atuação multiprofissional, construção do TCLE para os pacientes (de acordo com as recomendações das diretrizes) e criação de reuniões multiprofissionais para discussão de casos. Nesse sentido, a possibilidade de uma atuação multiprofissional emerge de uma visão ampliada, valorizando a importância da existência de espaços de discussão e possam permitir a construção de consensos entre os profissionais (SILVA; PEDUZZI, 2011).

3.5 Aplicação à realidade: planejando ações viáveis com possibilidade de mudanças 


\section{Programa de Pós-Graduação \\ em Ensino na Saúde - MEPES/UFG \\ DOSSIÊ \\ ENSINO NA SAÚDE}

ITINERARIUS

REFLECIONIS

V.17, N.01, 2021. ISSN: 1807-9342

O grupo de trabalho em questão apresenta uma relação afetiva entre si, construída no contato permanente no trabalho e demonstrou atitudes colaborativas em relação ao ideal de busca por melhorias na assistência e serviço de TRS, apesar de angústias e entraves citados como intervenientes no processo de aplicação da realidade.

Foi aplicada a ferramenta de gestão $5 \mathrm{~W} 3 \mathrm{H}$, representada em forma de esquema na Figura 3. Com isso, foi possível a estruturação da etapa de aplicação à realidade neste estudo, por meio de uma tabela, composta pelas três propostas mais viáveis, elencadas na etapa anterior, o que facilitou a elaboração de um plano de ação com possibilidades e alternativas que permitissem produzir mudanças no cotidiano do trabalho. Essa ferramenta proporciona trabalhar com atividades definidas e que devem ser desenvolvidas a partir da identificação e priorização de ações, estabelecendo o que deve ser feito (what?) e porque (why?), quem o fará (who?), em que período de tempo (when?) e onde na instituição (where?), além de como a atividade deve ser desenvolvida (how?), e sempre que possível o seu custo (how much?) e para acompanhamento de resultados o estabelecimento de indicadores (how measure?) (CALEMAN et al, 2016).

Figura 3: Esquema representativo da ferramenta 5W3H.

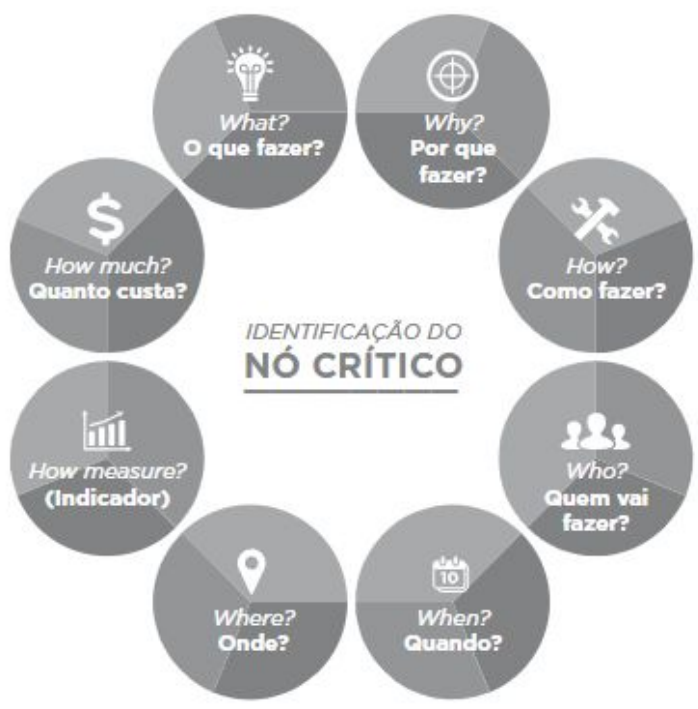

Fonte: Caleman et al. (2016, p. 36). 


\section{Programa de Pós-Graduação \\ em Ensino na Saúde - MEPES/UFG \\ DOSSIÊ \\ ENSINO NA SAÚDE}

ITINERARIUS

REFLECIONIS

V.17, N.01, 2021. ISSN: 1807-9342

Algumas ações, foram consideradas de maior viabilidade por depender exclusivamente dos participantes que as planejaram. Outras, ao contrário, dependiam de variáveis controladas por terceiros, tais como gestores da instituição, o que dificultaria a execução das ações.

A aproximação concreta da realidade permitida pela MP responde às necessidades de profissionais que pensem além da sua área de formação, considerando demandas da assistência aos usuários do Sistema Único de Saúde. Dessa forma, nessa etapa os participantes tomam e executam decisões permitindo a consciência do seu compromisso com a transformação da realidade com os serviços no qual estão inseridos, sendo o momento de planejamento e execução, além de um compromisso social (VILLARDI; CYRINO; BERBEL; 2015).

Sabe-se que no uso da MP não existe um controle absoluto dos resultados pelos facilitadores em termos de conhecimentos, sendo importante a sua postura em relação ao processo educativo de formação profissional e aos problemas relativos a situação em estudo. Dessa forma a MP diferencia-se de outras metodologias problematizadoras por permitir a problematização da realidade com um recorte feito pelos próprios educandos e retornar após obtenção de novas informações com o intuito de transformá-la em algum grau (COLOMBO; BERBEL, 2007; VIEIRA; PANÚNCIO-PINTO, 2015).

Vale acrescentar que a estrutura do método do Arco tendo a realidade como ponto de partida e chegada permite e representa movimentos contínuos, onde após o estudo de um problema poderão surgir outros desdobramentos, exigindo por exemplo contato com conteúdos que não haviam sido previstos anteriormente, mas que se fazem necessários para a compreensão e solução do problema; além de permitir a origem de novos arcos (VIEIRA; PANÚNCIO-PINTO, 2015).

\section{CONSIDERAÇÕES FINAIS}




\section{Programa de Pós-Graduação \\ em Ensino na Saúde - MEPES/UFG \\ DOSSIÊ \\ ENSINO NA SAÚDE}

ITINERARIUS

REFLECIONIS

V.17, N.01, 2021. ISSN: 1807-9342

Esse estudo apresenta como limitações a realização em uma unidade hospitalar com dificuldades relacionadas a horário e escala de trabalho dos participantes, assim como a restrição a um único serviço de TRS. Logo, sugere-se novos estudos com maior número de participantes além de ampliação para outros serviços que ofertam assistência a pacientes portadores de DRC em terapia dialítica com a utilização de ferramentas de transformação da realidade. Por outro lado, a participação de diferentes profissionais e o espaço de discussão contribuiu com a troca e o enfrentamento dos problemas desse serviço, sendo importante a criação de mecanismos que estimulem o envolvimento da equipe multiprofissional.

A vivência da EPS enquanto prática transformadora, alicerçada na MP, permanece sendo um desafio diante da dificuldade de associar a realidade cotidiana dos serviços com espaços de discussão coletiva em busca da renovação de processos de trabalho. Entretanto, essa estratégia em consonância com as recomendações da PNEPS propiciou condições de aprendizado significativo baseado em uma situação concreta do ambiente laboral.

A partir das experiências vividas na aplicação da MP, cabe destacar que a forma de abordar os saberes prévios dos profissionais envolvidos e a atitude problematizadora, visou a produção de uma educação mais crítica e reflexiva, ao invés de reprodutora e tecnicista. Porém, independente da utilização da MP ou de outras metodologias ativas de ensino-aprendizagem, o seu uso isolado não garante uma educação transformadora, sendo que a resistência a mudanças representa um forte obstáculo a ser enfrentado.

O poder decisório dos participantes na escolha do problema a ser trabalhado nos encontros e possibilidade de superação desse construída no decorrer da estratégia, foram aspectos que favoreceram a tomada de decisão coletiva na unidade com base na viabilidade das propostas. Além disso, a experiência de vivenciar e refletir sobre a MP, permitiu que pudessem avançar repensando e reconstruindo suas próprias práticas para melhoria dos serviços oferecidos, pois quanto mais ativo, crítico e reflexivo for o processo, maiores serão as chances de promoção de mudanças na realidade e contexto do trabalho.

\section{REFERÊNCIAS BIBLIOGRÁFICAS}




\section{Programa de Pós-Graduação \\ em Ensino na Saúde - MEPES/UFG \\ DOSSIÊ \\ ENSINO NA SAÚDE}

ITINERARIUS

REFLECIONIS

V.17, N.01, 2021. ISSN: 1807-9342

ANVISA. Agência Nacional de Vigilância Sanitária. Portaria $\mathbf{n}^{\mathbf{0}}$ 389, de 13 de março de 2014. Define os critérios para a organização da linha de cuidado da pessoa com Doença Renal Crônica (DRC) e institui incentivo financeiro de custeio destinado ao cuidado ambulatorial pré-dialítico. Brasília, DF: ANVISA, 2014.

ANVISA. Agência Nacional de Vigilância Sanitária. Resolução RDC n $^{\circ}$ 154, de 15 de junho de 2004. Estabelece o regulamento técnico para o funcionamento dos serviços de diálise. Brasília, DF: ANVISA, 2004.

BARDIN, Laurence. Análise de conteúdo. São Paulo: Edição 70; 2016.

BASTOS, M. G.; KIRSZTAJN, G. M. Doença Renal Crônica: importância do diagnóstico precoce, encaminhamento imediato e abordagem interdisciplinar estruturada para a melhora do desfecho em pacientes ainda não submetidos à diálise. Jornal Brasileiro de Nefrologia, São Paulo, v. 33, n. 1, p. 93-108, 2011.

BERBEL, N. A. N. A problematização e a aprendizagem baseada em problemas: diferentes termou ou diferentes caminhos? Interface: Comunicação, Saúde, Educação, Botucatu, v. 2, n. 2, p. 139-154, fev. 1998.

BERBEL, N. A. N. A Metodologia da Problematização em três versões no contexto da didática e da formação de professores. Revista Diálogo Educional, Curitiba, v. 12, n. 35, p. 103-120, jan./abr. 2012.

BERBEL, N. A. N.; GAMBOA, S. A. S.A Metodologia da Problematização com o Arco de Maguerez: uma perspectiva teórica e epistemológica. Filosofia e Educação (online), Campinas, v. 3, n. 2, p. 264-287, out. 2011/mar. 2012.

BORDENAVE, J. D.; PEREIRA, A. M. Estratégias de ensino aprendizagem. 4. ed. Petrópolis: Vozes, 1982.

BRASIL. Portaria n. 198 GM/MS, de 13 de fevereiro de 2004. Política Nacional de Educação Permanente em Saúde. Diário Oficial da República Federativa do Brasil, Brasília, 16 fev. 2004. Seção 1, p. 37-41.

BRASIL. Conselho Nacional de Saúde. Resolução CNS 466/2012. Diretrizes e Normas Regulamentadora de Pesquisa Envolvendo Seres Humanos. Brasília, DF: CNS, 2012.

CALEMAN, G.; LIMA, V. V.; OLIVEIRA, M. S. DE; SILVA, S. F. DA; MASSARO; A.; GOMES, R.; PEREIRA, S. M. DOS S. F.; VECCINA NETO, G.; COELHO, I.; 


\section{Programa de Pós-Graduação \\ em Ensino na Saúde - MEPES/UFG \\ DOSSIÊ \\ ENSINO NA SAÚDE}

ITINERARIUS

REFLECIONIS

V.17, N.01, 2021. ISSN: 1807-9342

SCARAZATTI, G. L.; SOEIRO, E.; RIBEIRO, E. C. O.; PADILHA, R. DE Q.; SCHIESARI, L. M. C.; RAMOS, L.; PETTA, H. R.; OLIVEIRA, J. M. DE; BIANCO, L.; CARVALHO, C. N. Projeto aplicativo: termos de referência. São Paulo: Ministério da Saúde; Instituto Sírio-Libanês de Ensino e Pesquisa, 2016.

CAMPOS, K. F. C.; SENA, R. R. DE; SILVA, K. L. Educação Permanente nos serviços de saúde. Escola Anna Nery, v. 21, n. 4, p. 1-10, 2017.

CECCIM, R. B.; CYRINO, E. G. O sistema de saúde e as práticas educativas na formação dos estudantes da área. In: . (Org.). Formação profissional em saúde e protagonismo dos estudantes: percursos na formação pelo trabalho. $1^{\text {a }}$ ed. Porto Alegre: Rede Unida, 2017, v. 1, p. 4-26.

COlOMBO, A. A.; BERBEL, N. A. N. A Metodologia da Problematização com o Arco de Maguerez e sua relação com os saberes de professores. Semina: Ciências Sociais e Humanas, Londrina, v. 28, n. 2, p. 121-146, jul./dez. 2007.

CONTERNO, S. DE F. R.; LOPES, R. E. Pressupostos pedagógicos das atuais propostas de formação superior em saúde no Brasil: origens históricas e fundamentos teóricos. Avaliação: Revista da Avaliação da Educação Superior (Campinas), Sorocaba, v. 21, n. 3, p. 993-1016, nov. 2016.

DE PAUlA, C. C.; PADOIN, S. M. DE M.; NIETSCHE, E. A.; BRUM, C. N. DE; RODRIGUES, A. P. Vulnerabilidade à infecção pelo HIV no adolescer: educação em saúde mediada pela metodologia da problematização. Adolescência e Saúde, v. 10, n. 1, p. 63-67, 2013.

FUJITA, J. A. L. DA M.; CARMONA, E. V.; SHIMO, A. K. K.; MECENA, E. H. DE. Uso da metodologia da problematização com o Arco de Maguerez no ensino sobre brinquedo terapêutico. Revista Portuguesa de Educação, v. 29, n. 1, p. 229-258, 2016.

GIGANTE, R. L.; CAMPOS, G. W. DE S. Política de formação e Educação Permanente em Saúde no Brasil: bases legais e referências teóricas. Trabalho, Educação e Saúde, Rio de Janeiro, v. 14, n. 3, p. 747-763, set./dez. 2016.

GOLDSTEIN, M.; YASSA, T.; DACOURIS, N.; MCFARLANE, P. Multidisciplinary predialysis care and morbidity and mortality of patientes on dialysis. American Journal of Kidney Diseases, v. 44, n. 4, p. 706-714, out. 2004. Disponível em: $<$ https://www.ajkd.org/article/S0272-6386(04)00940-0/pdf $>$. Acesso em: out. 2018.

HAMILTON, G.; SESSOMS, M.. Improving workflow in the dialysis clinic (Part 1). Nephrology News \& Issues, v. 25, n. 11, p. 32-40, 2011. 


\section{Programa de Pós-Graduação \\ em Ensino na Saúde - MEPES/UFG \\ DOSSIÊ \\ ENSINO NA SAÚDE}

ITINERARIUS

REFLECIONIS

V.17, N.01, 2021. ISSN: 1807-9342

HEMMELGARN, B. R.; MANNS, B. J.; ZHANG, J.; TONELLI, M.; KLARENBACH, S.; WALSH, M.; CULLETON, B. F. Association between multidisciplinary care and survival for elderly patients with Chronic Kidney Disease. Journal of the American Society of Nephrology, v. 18, p. 993-999, 2007.

MELO, M. C.; BOECKMANN, L. M. M.; MOURA, A. S. DE; GUILHEM, D. Aprendizagem baseada na problematização: utilizando o Arco de Maguerez na graduação de enfermagem. Revista Eletrônica Gestão \& Saúde, Brasília, v. 07, n. 1, p. 247-259, 2016.

MEHRY, E. E. Educação Permanente em movimento - uma política de reconhecimento e cooperação, ativando os encontros do cotidiano no mundo do trabalho em saúde, questões para gestores, trabalhadores e quem mais quiser se ver nisso. Saúde em Redes, Porto Alegre, v. 1, n. 1, p. 7-14, 2015.

PRADO, M. L. DO; VELHO, M. B.; ESPÍNDOLA, D. S.; SOBRINHO, S. H.; BACKES, V. M. S. Arco de Charles Maguerez: refletindo estratégias de metodologia ativa na formação de profissionais de saúde. Escola Anna Nery, Rio de Janeiro, v. 16, n. 1, p. 172-177, jan./mar. 2012.

SESSOMS, M.; HAMILTON, G. Improving workflow in the dialysis clinic (Part 2). Nephrology News \& Issues, v. 25, n. 12, p. 27-36, 2011.

SILVA, J. A. M.; PEDUZZI, M. Educação no trabalho na atenção primária à saúde: interfaces entre a educação permanente em saúde e o agir comunicativo. Saúde e Sociedade, v. 20, n. 4, p. 1018-1032, 2011.

SILVA, J. C. ; MORAIS, E. R. DE; FIGUEIREDO, M. DO L.F.; TYRRELL, M. A. R. Pesquisa-ação: concepções e aplicabilidade nos estudos em Enfermagem. Revista Brasileira de Enfermagem, Brasília, v. 64, n. 3, p. 592-595, mai.jun. 2011.

SINGH, A.; KARI, J. Manejo dos estágios 4 e 5 da doença renal. In: DAUGIRDAS, J. T.; BLAKE, P. G.; ING, T. S. (Org.). Manual de Diálise. 5. ed. Rio de Janeiro: Guanabara Koogan, 2016. cap. 2, p. 18-24.

SOUSA, M. R. G.; SILVA, A. E. B. DE C.; BEZERRA, A. L. Q.; FREITAS, J. S. DE; MIASSO, A. I. Eventos adversos em hemodiálise: relatos de profissionais de enfermagem. Revista da Escola de Enfermagem da USP, São Paulo, v. 47, n. 1, p. 76-83, 2013.

THOMÉ, F. S.; SESSO, R. C.; LOPES, A. A.; LUGON, J. R.; MARTINS, C. T. Inquérito Brasileiro de Diálise Crônica 2017. Jornal Brasileiro de Nefrologia, v. 41, n. 2, p. 208-214, 2019. 
TONG, A.; SAINSBURY, P.; CRAIG, J. Consolidated criteria for reporting qualitative research (COREQ): A 32-item checklist for interviews and focus groups. International Journal for Quality in Health Care, v. 19, n. 6, p. 349-357, 2007.

TRIPP, D. Pesquisa-ação: uma introdução metodológica. Educação e Pesquisa, São Paulo, v. 31, n. 3, p. 443-466, set./dez. 2005.

VIEIRA, M. N. C. M.; PANÚNCIO-PINTO, M. P. A Metodologia da Problematização (MP) como estratégia de integração ensino-serviço em cursos de graduação na área da saúde. Revista da Faculdade de Medicina de Ribeirão Preto, Ribeirão Preto, v. 48, n. 3, p. 241-248, 2015.

VILlARDI, M. L.; CYRINO, E. G.; BERBEL, N. A. N. A Metodologia da Problematização no ensino em saúde: suas etapas e possibilidades. In: . (Org.). A problematização em educação em saúde: percepção dos professores tutores e alunos. São Paulo: Cultura Acadêmica, 2015, cap. 2, p. 45-52.

Webster, A. C.; NAGLER, E. V.; MORTON, R. L.; MASSON, P. Chronic Kidney Disease. The Lancet, v. 389, n. 10075, p. 1238-1252, mar. 2017.

WERNECK, M. A. F.; FARIA, H. P. DE; CAMPOS, K. F. C. Protocolos de cuidados à saúde e de organização do serviço. Belo Horizonte: Nescon/UFMG, Coopmed, 2009. 